\title{
Escape-limited maximum energy at perpendicular shocks in the interstellar magnetic field
}

\author{
Shoma F. Kamijima ${ }^{a, *}$ and Yutaka Ohira ${ }^{a}$ \\ ${ }^{a}$ Department of Earth and Planetary Science, The University of Tokyo, 7-3-1 Hongo, Bunkyo-ku, Tokyo \\ 113-0033, Japan \\ E-mail: s.kamijima@eps.s.u-tokyo.ac.jp, y.ohira@eps.s.u-tokyo.ac.jp
}

\begin{abstract}
We investigate the maximum energy limited by the escape from a perpendicular shock region of a spherical shock in the interstellar medium (ISM), and the size of the rapid acceleration region. The perpendicular shock in supernova remnants (SNRs) has been expected to be PeVatrons without a magnetic field amplification in the upstream region. We perform test particle simulations, showing that the escape-limited maximum energy in the perpendicular shock is about a few $10 \mathrm{TeV}$ for the typical type Ia SNRs.
\end{abstract}

$37^{\text {th }}$ International Cosmic Ray Conference (ICRC 2021)

July 12th-23rd, 2021

Online - Berlin, Germany

\footnotetext{
*Presenter
} 


\section{Introduction}

Supernova remnants (SNRs) are the plausible candidate of a PeVatron, and are believed to be the source of Galactic cosmic rays (CRs). The diffusive shock acceleration (DSA) is the probable mechanism to accelerate $\mathrm{CRs}$ to PeV scale [1-4]. The HAWC experiment reported PeVatron candidates in our galaxy [5-7]. An evidence of PeVatrons near the Galactic center was found by the HESS observation [8]. The Tibet AS $\gamma$ expetiment observed sub-PeV diffuse gamma rays and found a potential PeVatron SNR in our galaxy [9, 10]. The LHAASO expetiment recently reported some PeVatron candidates in our galaxy [11-13]. However, we have not fully understood what types of SNRs can be PeVatrons. It is claimed that DSA in SNRs cannot accelerate CRs to PeV in the interstellar magnetic field, which is about a few $\mu \mathrm{G}$. To accelerate CRs to PeV in SNRs, a magnetic field amplification to about $100 \mu \mathrm{G}$ in the upstream region is needed. Some magnetic amplification mechanisms have been proposed and studied by several simulations [14-17]. However, it is still unknown whether or not a sufficient magnetic field amplification in the upstream region can be realized.

It was suggested that a perpendicular shock can rapidly accelerate $\mathrm{CRs}$ to $\mathrm{PeV}$ without an upstream magnetic field amplification. In addition, it was claimed that the momentum spectrum in the perpendicular shock becomes softer than that of the standard DSA prediction, $d N / d p \propto p^{-2}$, when the magnetic turbulence is weak both in the upstream and downstream regions to realize the rapid acceleration [18]. However, a strong downstream turbulence is recently reported by some observations and simulations [21-24]. Thus, we considered the weak upstream turbulence and the strong downstream turbulence. Under this condition, the rapid acceleration and the canonical spectrum are simultaneously realized [19]. However, it is still unknown the size of the acceleration region that CRs are rapidly accelerated.

It was recently pointed out that the maximum energy of CRs can be limited by the escape process from accelerators [25-27]. In addition, the spectral index of escaped CRs is determined by the time evolution of the escape-limited maximum energy [27]. Therefore, the escape process and the escape-limited maximum energy are vital issues to understand the maximum energy and the observed energy spectrum of CRs. To reveal the escape process and the escape-limited maximum energy, previous studies applied the diffusion approximation that neglects the gyro motion in the magnetic field. However, the gyro motion is important to realize the rapid acceleration in perpendicular shocks [19]. Thus, the escape process from the perpendicular shock region and the escape-limited maximum energy have not been investigated without the diffusion approximation. In this work, we first investigate the escape-limited maximum energy at the perpendicular shock for a spherical shock wave in the interstellar magnetic field.

In this work, we consider type Ia SNRs because surroundings of typical type Ia SNRs are the interstellar medium (ISM). Shock waves of core collapse SNRs also propagates the ISM after about $10^{4}$ years since their supernova explosions. However, for $t \gtrsim 10^{4} \mathrm{yr}$, the radiation cooling in the shocked ISM region becomes important [29]. In addition, the shock velocity for $t \gtrsim 10^{4} \mathrm{yr}$ is too slow to accelerate CRs to PeV. Therefore, in this work, we consider only type Ia SNRs. 


\section{Simulation}

The shock evolution of type Ia SNRs is given by [28]. Here, we assume the explosion energy, $E_{\mathrm{SN}}=10^{51} \mathrm{erg}$, the ejecta mass, $M_{\mathrm{ej}}=1 M_{\odot}$, and the mass density, $\rho=1.67 \times 10^{-24} \mathrm{~g} \mathrm{~cm}^{-3}$. The upstream magnetic field consists of the uniform magnetic field and a turbulent magnetic field, $\vec{B}_{1}=\vec{B}_{0}+\delta \vec{B}$. The uniform magnetic field strength, $\left|\vec{B}_{0}\right|$, is set to be $3 \mu \mathrm{G}$. The turbulent magnetic field, $\delta \vec{B}$, is represented by the summation of static plane waves [31]. The injection scale, the amplitude, and the spectrum of the turbulence are set to be 1 or $100 \mathrm{pc}$, the isotropic Kolmogorov spectrum, and $|\delta \vec{B}|=\left|\vec{B}_{0}\right|$.

In this work, the downstream magnetic field strength, $B_{2}$, is assumed to be amplified from the shock compressed value. We assumed that a fraction of the energy flux of the upstream kinetic energy is converted to the downstream magnetic energy flux.

$$
B_{2}=\sqrt{16 \pi \varepsilon_{B} \rho} u_{\mathrm{sh}},
$$

where $\varepsilon_{B}$ is the conversion fraction, and $u_{\mathrm{sh}}$ is the shock velocity in the ISM rest frame. In this work, we use $\varepsilon_{B}=10^{-2}$. As for the velocity profile inside the spherical shock, we use an approximate solution of $u_{2}(r)=\left(3 u_{\mathrm{sh}} / 4\right)\left(r / R_{\mathrm{sh}}\right)$, where $r$ is the radial distance from the explosion center [30].

We use different methods to solve the particle motion in the upstream and downstream regions. In the downstream region, we solve random work by the Monte-Carlo method because we assume the highly turbulent downstream magnetic field, and the isotropic particle scattering in local fluid rest frame. As for the downstream diffusion, we set the Bohm limit in the downstream magnetic field. On the other hand, in the upstream region, we solve the particle trajectory in the upstream magnetic field, $\vec{B}$. We use the Bunemann-Boris method to solve the equation of motion for upstream particles [32]. Particles are impulsively injected into the whole shock surface at $t_{\text {inj }}=$ $40,100,200,660,2000,6600$, and $20000 \mathrm{yr}$. The energy of injected particles at $t_{\mathrm{inj}}=20000 \mathrm{yr}$ is $100 \mathrm{GeV}$, and $1 \mathrm{TeV}$ for the other injection times. The particle splitting method is applied to improve statistics of the number of high energy particles.

\section{Simulation results}

In Figure 1, we show the maximum energy of particles. The horizontal axis and the vertical axis show the SNR age and the maximum energy of particles, respectively. The black dotted line is the age-limited maximum energy, $E_{\text {max age }}$, given by the condition that the acceleration time, $t_{\text {acc }}$ (see Equation (5) in [19]), is equal to the SNR age, $t_{\text {age. }}$ The age-limited maximum energy in this work is

$$
E_{\text {max }, \text { age }}=\left(\frac{u_{\mathrm{sh}}}{v}\right)^{2}\left(\frac{B_{2}}{B_{1}}\right)\left\{\pi\left(\frac{u_{\mathrm{sh}}}{v}\right)\left(\frac{B_{2}}{B_{1}}\right)+\frac{16}{3}\right\}^{-1} Z e B_{1} v t_{\mathrm{age}},
$$

where $v$ is the particle velocity, $Z e$ is the particle charge. In our acceleration model, we assume that the upstream and downstream particle motions are the gyration and the Bohm diffusion, respectively. The blue open circles and squares are simulation results for the turbulent magnetic field with the injection scale of $1 \mathrm{pc}$ and $100 \mathrm{pc}$, respectively. Simulation results are extracted as follows. First, we make the momentum spectrum of escaping CRs, $p^{2} d N / d p$. The peak momentum around the 


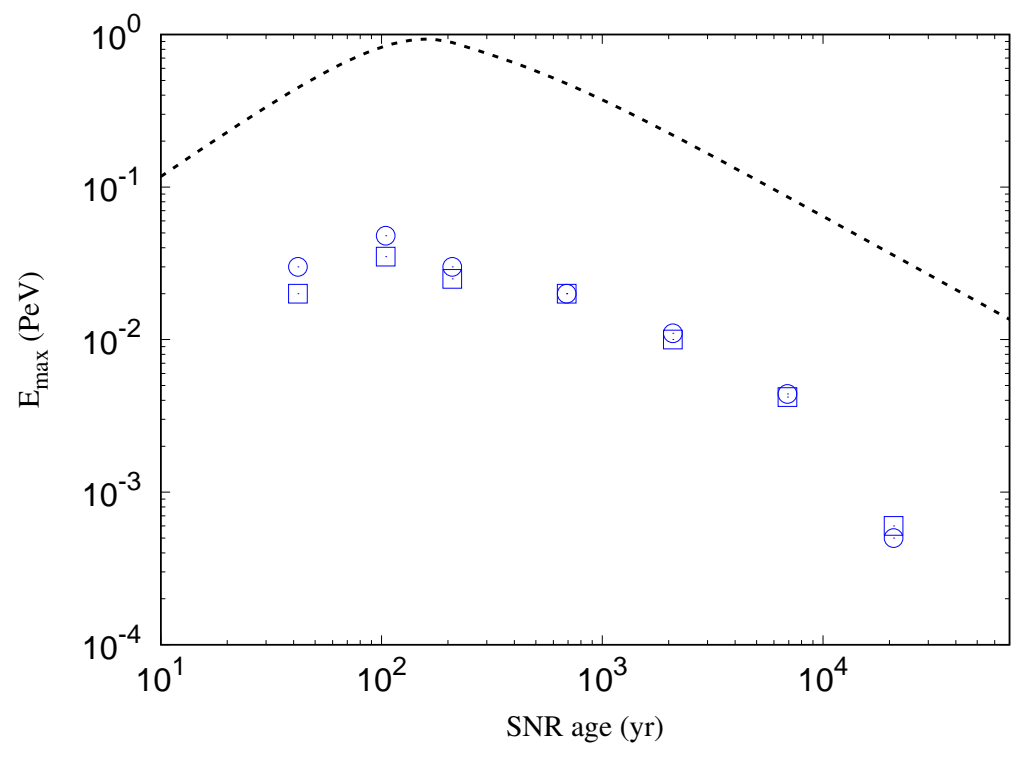

Figure 1: Maximum energy of particles accelerated in the perpendicular shock of type Ia SNRs. The black dotted line are the age-limited maximum energy (Equation (2)). The blue open circles and squares are simulation results for the turbulent magnetic field with the injection scale of $1 \mathrm{pc}$ and $100 \mathrm{pc}$, respectively.

cut-off is estimated at every some time steps. We regard the particles as escaping particles when particles move more than $10 \%$ of the shock radius away from the shock surface. Next, we see the time evolution of the peak momentum of $p^{2} d N / d p$ for escaping CRs. We identify the maximum value of the peak momentum as the escape-limited maximum energy. As one can see, simulation results are always at least ten times smaller than the age-limited maximum energy. The results for the turbulent magnetic field with the injection scale of $1 \mathrm{pc}$ do not change significantly from that for $100 \mathrm{pc}$. Thus, the perpendicular shock in type Ia SNRs in the ISM cannot accelerate CRs to PeV. Theoretical estimations for the escape-limited maximum energy and the size of the acceleration are shown in [20]

Figure 2 shows the time evolution of the particle distribution projected on the $x z$ plane for the case that the injection scale is set to be $1 \mathrm{pc}$. The particle position is normalized by the shock radius at $t_{\text {inj }}=40 \mathrm{yr}$. The black circle is the shock front. The points and color show the particle position and the particle energy, respectively. Particles injected into the equator are rapidly accelerated at the perpendicular shock region, and escape from the perpendicular shock region while diffusing along the upstream magnetic field line. Furthermore, even if particles are injected into the equator, particles concentrate on the polar region [33].

\section{Discussion}

For core collapse supernovae, the initial progenitor mass is stripped by the stellar wind before the explosion. Thus, the explosion of core collapse supernovae occurs in the circumstellar medium (CSM). It is expected that the magnetic field configuration in the CSM is the Parker spiral structure. 

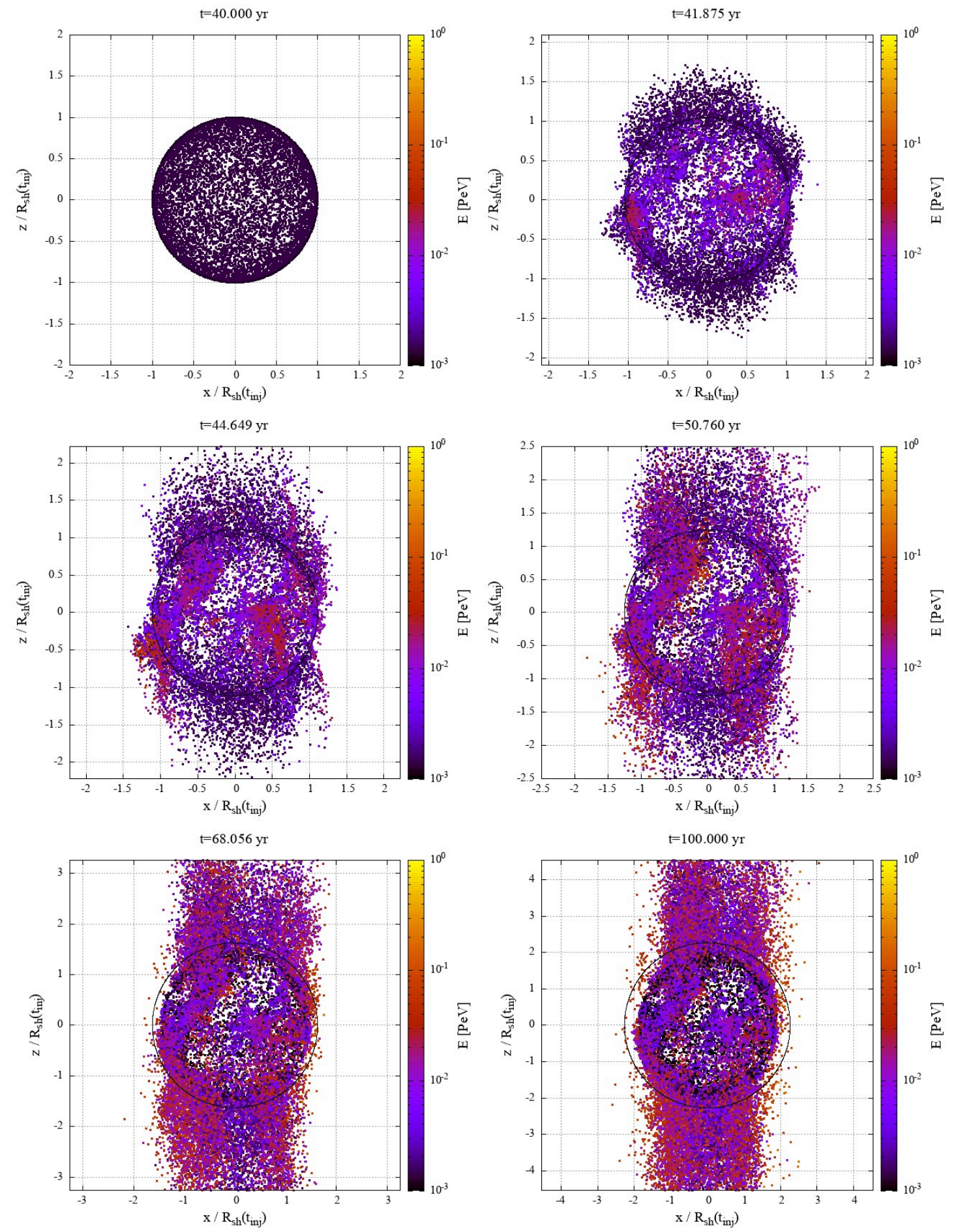

Figure 2: The time evolution of the particle distribution projected to $x z$ plane for the case that the injection scale is set to be $1 \mathrm{pc}$. The particle position is normalized by the shock radius at $t_{\mathrm{inj}}=40 \mathrm{yr}$. The black circle is the shock front. The color shows the particle energy. 
Then, large areas of SNR shocks in the CSM become perpendicular shocks, so that CRs could be accelerated to PeV. In the future, we are going to investigate the $\mathrm{CR}$ acceleration and the escape process in the Parker spiral magnetic field.

\section{Summary}

In this work, we first investigate the CR escape process from the perpendicular shock of the spherical shock in the ISM magnetic field. The perpendicular shock region in SNRs is expected to be PeVatron without an upstream magnetic field amplification, however, the escape-limited maximum energy is still unknown. We performed test particle simulations to reveal the escape-limited maximum energy. We showed that the escape-limited maximum energy is about a few $10 \mathrm{TeV}$. Thus, we conclude that the maximum energy is not limited by the finite age, but always limited by the escape process. The perpendicular shock of type Ia SNRs in the ISM cannot accelerate CRs to $\mathrm{PeV}$.

\section{Acknowledgments}

We thank M. Hoshino and T. Amano for valuable comments. Numerical computations were carried out on Cray XC50 at Center for Computational Astrophysics, National Astronomical Observatory of Japan. S.K. is supported by International Graduate Program for Excellence in Earth-Space Science (IGPEES), The University of Tokyo. Y.O. is supported by JSPS KAKENHI Grant Number JP19H01893, and by Leading Initiative for Excellent Young Researchers, MEXT, Japan.

\section{References}

[1] W. I. Axford et al., Proc. 15th Int. Cosmic Ray Conf. 11, 132 (1977).

[2] G. F. Krymsky, Sov. Phys. Dokl. 23, 327 (1977).

[3] A. R. Bell, Mon. Not. R. Astron. Soc. 182, 147 (1978).

[4] R. D. Blandford and J. P. Ostriker, Astrophys. J. 221, L29 (1978).

[5] A. Albert et al. (HAWC Collaboration), Astrophys. J. Lett. 896, L29 (2020).

[6] A. Albert et al. (HAWC Collaboration), Astrophys. J. Lett. 907, L30 (2021).

[7] A. Albert et al. (HAWC Collaboration), Astrophys. J. Lett. 911, L27 (2021).

[8] A. Abramowski et al. (H.E.S.S. Collaboration), Nature(London) 531, 476 (2016).

[9] M., Bao et al. (The Tibet AS $\gamma$ Collaboration), Nat. Astron. 5, 460 (2021).

[10] M. Amenomori et al. (The Tibet AS $\gamma$ Collaboration), Phys. Rev. Lett. 126, 141101 (2021)

[11] Zhen Cao et al., Nat. Astron. 594, 33 (2021). 
[12] Zhen Cao et al. (LHAASO Collaboration), Discovery of the Ultra-high energy gamma-ray source LHAASO J2108+5157, arXiv:2106.09865.

[13] F. Aharonian et al. (LHAASO Collaboration), Phys. Rev. Lett. 126, 241103 (2021).

[14] J. Niemiec et al., Astrophys. J. 684, 1174 (2008).

[15] V. N. Zirakashvili and V. S. Ptuskin, Astrophys. J. 678, 939 (2008).

[16] Y. Ohira et al., Astrophys. J. 698, 445 (2009).

[17] M. A. Riquelme and A. Spitkovsky, Astrophys. J. 694, 626 (2009).

[18] M. Takamoto and J. G. Kirk, Astrophys. J. 809, 29 (2015).

[19] S. F. Kamijima, Y. Ohira, and R. Yamazaki, Astrophys. J. 897, 116 (2020).

[20] S. F. Kamijima and Y. Ohira, Escape of cosmic rays from perpendicular shocks in the interstellar magnetic field, arXiv:2107.05820.

[21] J. Giacalone and J. R. Jokipii, Astrophys. J. Lett. 663, L41 (2007).

[22] T. Inoue, R. Yamazaki and S. Inutsuka, Astrophys. J. 695825 (2009).

[23] Y. Ohira, Astrophys. J. 817, 137 (2016).

[24] Y. Ohira and R. Yamazaki, J. High Energy Astrophys. 13-14, 17 (2017).

[25] V. S. Ptuskin and V. N. Zirakashvili, Astron. Astrophys. 403, 1 (2003).

[26] V. S. Ptuskin and V. N. Zirakashvili, Astron. Astrophys. 429, 755 (2005).

[27] Y. Ohira, K. Murase and R. Yamazaki, Astron. Astrophys. 513, 17 (2010).

[28] C. F. McKee and J. K. Truelove, Phys. Rep. 256, 157 (1995).

[29] J. K. Truelove and C. F. McKee, Astrophys. J. 120, 299 (1999).

[30] Y. Ohira, S. Kisaka and R. Yamazaki, Mon. Not. R. Astron. Soc. 00, 1 (2018).

[31] M. Hussein, and A. Shalchi, Astrophys. J. 785, 31 (2014).

[32] C. K. Birdsall and A. B. Langdon, Plasma Physics via Computer Simulation, The Adam Hilger Series on Plasma Physics(Adam Hilger, Bristol) (1991).

[33] J. Giacalone, Astrophys. J. 848, 123 (2017). 